\title{
CsgE is a curli secretion specificity factor that prevents amyloid fibre aggregation
}

Ashley A. Nenninger, ${ }^{1}$ Lloyd S. Robinson, ${ }^{1}$ Neal D. Hammer, ${ }^{2}$ Elisabeth Ashman Epstein, ${ }^{2}$ Matthew P. Badtke, ${ }^{2}$ Scott J. Hultgren ${ }^{1 *}$ and Matthew R. Chapman ${ }^{2 *}$

${ }^{1}$ Department of Molecular Microbiology and Microbial Pathogenesis, Washington University School of Medicine, Campus Box 8230, 660 S. Euclid Avenue, St Louis, MO 63110, USA.

${ }^{2}$ Department of Molecular, Cellular and Developmental Biology, University of Michigan, Ann Arbor, 830 North University, Ann Arbor, MI 48109, USA.

\section{Summary}

Curli are extracellular amyloid fibres produced by Escherichia coli that are critical for biofilm formation and adhesion to biotic and abiotic surfaces. CsgA and CsgB are the major and minor curli subunits, respectively, while CsgE, CsgF and CsgG direct the extracellular localization and assembly of curli subunits into fibres. The secretion and stability of CsgA and CsgB are dependent on the outer membrane lipoprotein CsgG. Here, we identified functional interactions between CsgG and CsgE during curli secretion. We discovered that CsgG overexpression restored curli production to a csgE strain under curli-inducing conditions. In antibiotic sensitivity and protein secretion assays, CsgG expression alone allowed translocation of erythromycin and small periplasmic proteins across the outer membrane. Coexpression of CsgE with CsgG blocked non-specific protein and antibiotic passage across the outer membrane. However, CsgE did not block secretion of proteins containing a 22-amino-acid putative outer membrane secretion signal of CsgA (A22). Finally, using purified proteins, we found that CsgE prohibited the selfassembly of CsgA into amyloid fibres. Collectively, these data indicate that CsgE provides substrate specificity to the curli secretion pore CsgG, and acts directly on the secretion substrate CsgA to prevent premature subunit assembly.

Accepted 10 May, 2011. *For correspondence. E-mail chapmanm@ umich.edu; Tel. (+1) 734764 7592; Fax (+1) 7346470884.

\section{Introduction}

Curli represent a novel class of bacterial proteinaceous surface fibres that are produced by enteric bacteria such as Escherichia, Salmonella and Citrobacter (Zogaj et al., 2003). Curli promote biofilm formation (Vidal et al., 1998), as well as mediate binding to a variety of eukaryotic proteins including fibronectin and MHC class I molecules (Olsen et al., 1989; 1998). Curli share biochemical and structural properties with disease-associated fibres called amyloids (Chapman et al., 2002). Amyloids are characterized as unusually stable $\beta$-sheet rich fibrous protein aggregates that bind to the dyes Congo red (CR) and thioflavin T (Nilsson, 2004). Unlike disease-associated amyloid formation, which is the product of protein misfolding, curli assembly is regulated by a dedicated biosynthesis pathway (Romling et al., 1998; Chapman et al., 2002; Barnhart and Chapman, 2006). Therefore, the curli biogenesis pathway provides unique insight into how cells control amyloidogenesis.

The curli specific genes $(c s g)$ are present on the divergently transcribed $\operatorname{csg} B A C$ and $\operatorname{csg} D E F G$ operons (Hammar et al., 1995). CsgA and CsgB constitute the major and minor curli subunits respectively. The major fibre subunit CsgA interacts with the CsgB nucleator protein at the cell surface and assembles into a highly insoluble and aggregative amyloid fibre (Hammar et al., 1996). The transcriptional activator CsgD is required for expression of the $\operatorname{csg} B A C$ operon, while CsgC, CsgE, $\mathrm{CsgF}$ and CsgG comprise the curli subunit assembly machinery (Hammar et al., 1995; Loferer et al., 1997; Chapman et al., 2002; Robinson et al., 2006; Gibson et al., 2007; Nenninger et al., 2009; Salgado et al., 2011). The curli assembly machinery functions to restrict curli amyloid formation to the extracellular space, thus minimizing potential cytotoxic effects of intracellular amyloidogenesis. However, the mechanism(s) by which the cell produces and secretes amyloidogenic proteins while preventing their internal assembly has remained elusive.

The $30 \mathrm{kDa}$ lipoprotein CsgG localizes to the outer membrane and in vivo is absolutely required for curli subunit stability and fibre synthesis (Loferer et al., 1997; Robinson et al., 2006). CsgG has periplasmic and extracellular domains, and is spatially clustered in cells at sites 
of curli assembly (Epstein et al., 2009). Consistent with its proposed role as the secretion channel for curli subunits, CsgG forms an SDS-resistant pore-like multimer at the outer membrane and interacts with the mature $\mathrm{N}$-terminus of the fibre subunit CsgA (Loferer et al., 1997; Robinson et al., 2006). The small chaperone-like proteins CsgE and CsgF modulate the levels of CsgA (Chapman et al., 2002). CsgF is surface-exposed and requires CsgG for its stability and surface localization (Nenninger et al., 2009). Functionally, CsgF is critical for CsgB-mediated nucleation at the cell surface and thus a $c s g F$ mutant secretes CsgA away from cells (Chapman et al., 2002; Robinson et al., 2006; Hammer et al., 2007; Nenninger et al., 2009).

The role of CsgE in curli assembly is unclear, although cells lacking $\operatorname{csg} E$ display phenotypes similar to a $c s g G$ mutant strain. Like the $\operatorname{csg} G$ phenotype, deletion of $c s g E$ results in white colonies on $\mathrm{CR}$-indicator agar, and nearly undetectable levels of the CsgG-secreted proteins, CsgA and CsgF (assessed previously) and CsgB (assessed in this study) (Loferer et al., 1997; Chapman et al., 2002; Robinson et al., 2006; Nenninger et al., 2009). Unlike the csgG mutant, csgE bacteria can assemble a small amount of CsgA-reactive fibres, with a distinct curved morphology compared with the straight and rigid morphology of wildtype curli fibres (Chapman et al., 2002). Importantly, CsgG stability and localization to the outer membrane are unaffected in csgE cells (Epstein et al., 2009).

Since the $\operatorname{csg} G$ and $\operatorname{csg} E$ phenotypes closely resemble each other, we investigated the role of CsgE in CsgGmediated secretion. In this work we show that CsgE and CsgG function in the same pathway to support the stability and secretion of CsgA, CsgB and CsgF. Our data indicate that $\mathrm{CsgE}$ modulates the pore-like properties of $\mathrm{CsgG}$ by acting as a specificity factor in CsgG-mediated translocation. Furthermore, we found that CsgE can inhibit the polymerization of purified CsgA into fibres, suggesting a model for how E. coli can prohibit intracellular amyloid formation.

\section{Results}

\section{csgE phenotypes are suppressed by CsgG overexpression}

$\mathrm{CsgG}$ is required for the localization and stability of CsgB, CsgA and CsgF. Thus, csgG mutants do not assemble curli (Hammar et al., 1995; Loferer et al., 1997; Robinson et al., 2006; Nenninger et al., 2009). csgE mutant phenotypes resemble $\operatorname{csg} G$ mutant phenotypes in that they produce little to no detectable CsgA, CsgF or curli fibres (Chapman et al., 2002; Robinson et al., 2006; Nenninger et al., 2009). Therefore, we examined the possibility that increased CsgG expression alleviates the requirement for CsgE in fibre subunit secretion and assembly. To test this hypothesis, we expressed CsgG in the csgE mutant strain at low levels using a medium-copy plasmid containing the $\operatorname{csgBA}$ promoter (pLR1, see Table 1), and at high levels using a high-copy overexpression plasmid (pTrc99a). Strains were plated on YESCA agar supplemented with the amyloid-binding dye $\mathrm{CR}$, which provides a measure of curli production. As shown in Fig. 1A, csgE mutant bacteria without vector or with the empty vectors pLR1 or pTrc99a (csgE, csgE/v and $c s g E / \mathrm{v}^{+}$respectively) were white on a CR-indicator plate as compared with the wildtype strain. CR binding was restored to near wild-type levels in the $c s g E$ mutant strain by low-level expression of CsgE (Fig. 1A, csgE/pE). Similarly, overexpression of CsgG restored wild-type levels of $C R$ binding to the $\operatorname{csg} E$ mutant (Fig. 1A, csgE/pG ${ }^{+}$). When CsgG was expressed at low levels, however, little CR binding was observed, suggesting high levels of $\mathrm{CsgG}$ expression were required to overcome the $\operatorname{csg} E$ mutant phenotype (Fig. 1A, csgE/ $p G)$. While low-level CsgE expression resulted in approximately wild-type levels of CR binding, overexpression of $\mathrm{CsgE}$ in the $\operatorname{csg} E$ strain resulted in reduced $\mathrm{CR}$ binding relative to both the wild-type and $c s g E / p E$ strains (Fig. 1A, compare wild-type, csgE/pE and $\left.c s g E / \mathrm{pE}^{+}\right)$. CsgF was unable to suppress the $c s g E$ mutant phenotype when expressed either at low or high levels (Fig. 1A, csgE/pF and $c s g E / \mathrm{pF}^{+}$respectively).

To investigate whether the CR binding phenotypes were due to curli production, we performed negative stain electron microscopy (EM) and Western blot analysis on each of the strains in Fig. 1A. By negative stain EM, the csgE mutant harbouring an empty vector displayed few extracellular fibres, most of which were characterized by a curved morphology (Fig. 1B, csgE/v), similar to what has been previously shown for the $c s g E$ mutant alone (Chapman et al., 2002). In contrast, the csgE mutant complemented with either low-level CsgE expression ( $\mathrm{pE}$ ) or high-level CsgG expression ( $\mathrm{pG}^{+}$) exhibited extracellular fibres of the same abundance and morphology as wild-type curli fibres, suggesting both plasmids were able to rescue the csgE mutant phenotype (Fig. 1B, csgE/pE and csgE/pG ${ }^{+}$).

By Western blot analysis of whole cell lysates, the csgE mutant contained dramatically decreased levels of CsgA, CsgF and CsgB (Fig. 1C, lane 2). Whole cell levels of CsgA, CsgB and CsgF were restored with low-level expression of $\mathrm{CsgE}$, as well as overexpression of $\mathrm{CsgG}$ (Fig. 1C, lanes 4 and 10). Overexpression of CsgE resulted in a phenotype similar to the $c s g E / p E$ strain: CsgA, CsgB and CsgF protein levels were largely restored to wild-type levels (Fig. $1 \mathrm{C}$, lane 8), fibres were observed by EM (Fig. 1B, csgE/pE') and very little CsgA was SDS-soluble, indicating that most or all of the CsgA produced by these strains was in an aggregated form (Fig. S1B). However, the $c s g E / p E^{+}$strain did not exhibit full complementation as measured by $\mathrm{CR}$ binding 
Table 1. Strains and plasmids used in this study.

\begin{tabular}{|c|c|c|}
\hline Strains/plasmids & Description & Source/reference \\
\hline \multicolumn{3}{|l|}{ Strains } \\
\hline MC4100 & F- $\operatorname{araD139} \Delta\left(\operatorname{argF-lac)U169} r s p L 150\left(\right.\right.$ Strr $\left.^{r}\right)$ relA1 flbB5301 deoC1 ptsF25 rbsR & Casadaban (1976) \\
\hline MHR480 & MC4100 $\Delta c s g E$ & Hammar et al. (1996) \\
\hline LSR11 & MC4100 $\Delta c s g$ & This study \\
\hline LSR35 & MC4100 csgEF::kan ${ }^{R}$ & This study \\
\hline NEB3016 & E. coli protein expression strain & New England Biolabs \\
\hline \multicolumn{3}{|l|}{ Plasmids } \\
\hline pBAD33 & arabinose-inducible expression vector & Guzman et al. (1995) \\
\hline pET11d & IPTG-inducible expression vector & Novagen \\
\hline pTrc99a & IPTG-inducible expression vector & Pharmacia Biotech \\
\hline pAN65 & $\begin{array}{l}\text { an ORF encoding the CsgA signal sequence fused to mature CpxP with a C-terminal 6-his tag } \\
\text { in pBAD33 (CsgAss-CpxP-his) }\end{array}$ & This study \\
\hline pAN66 & $\begin{array}{l}\text { an ORF encoding the CsgA signal sequence fused to mature CpxP with a C-terminal 6-his tag } \\
\text { in pTrc99a (CsgAss-CpxP-his) }\end{array}$ & This study \\
\hline pAN69 & $\begin{array}{l}\text { an ORF encoding the PapD signal sequence with a Gly-Thr linker (encoded by a ggtacc Kpnl } \\
\text { site) fused to mature CpxP with a C-teriminal 6-his tag, inserted into the Ncol-Pstl sites of } \\
\text { pLR92 (PapDss-[GT]-CpxP-his) }\end{array}$ & This study \\
\hline pAN70 & $\begin{array}{l}\text { an ORF encoding the PapD signal sequence fused to the mature N-terminus of CsgA (A22) } \\
\text { with a Gly-Thr linker (encoded by a ggtacc Kpnl site) fused to mature CpxP with a C-terminal } \\
\text { 6-his tag in pLR92 (PapDss-A22-[GT]-CpxP-his) }\end{array}$ & This study \\
\hline pAN87 & papD2-his cloned into the Kpnl-Pstl sites of pAN69 (PapDss-[GT]-PapD2-his) & This study \\
\hline pAN88 & papD2-his cloned into the Kpnl-Pstl sites of pAN70 (PapDss-A22-[GT]-PapD2-his) & This study \\
\hline pAN93 & the Ncol-Pstl fragment of pAN87 cloned into pTrc99a (PapDss-[GT]-PapD2-his) & This study \\
\hline pAN94 & the Ncol-Pstl fragment of pAN88 cloned into pTrc99a (PapDss-A22-[GT]-PapD2-his) & This study \\
\hline pLR1 & $\operatorname{csgBA}$ promoter cloned into the BamHI-Pstl sites of pACYC177 & Robinson et al. (2006) \\
\hline pLR42 & $\operatorname{csg} E$ inserted into the Kpnl-Pstl sites of pBAD33 & This study \\
\hline pLR50 & $\begin{array}{l}\text { an ORF encoding the CsgA signal sequence and mature N-terminus (A22) fused to mature } \\
\text { CpxP with a C-terminal 6-his tag inserted into the Kpnl-Pstl sites of pBAD33 } \\
\text { (CsgAss-A22-CpxP-his) }\end{array}$ & This study \\
\hline pLR51 & the CsgAss-A22-CpxP-his Ncol-Pstl fragment of pLR50 inserted into pLR1 & This study \\
\hline pLR58 & csgF-HA in pBAD33 & Robinson et al. (2006) \\
\hline pLR70 & The $\operatorname{csg} E$ Ncol-Pstl fragment of pLR42 inserted into pLR1 & This study \\
\hline pLR71 & $\operatorname{csg} E$ inserted into the Ncol-BamHI sites of pTrc99a & This study \\
\hline pLR73 & $\operatorname{csg} F$ in pLR1 & Nenninger et al. (2009) \\
\hline pLR74 & csgF inserted into the Ncol-Pstl sites of pTrc99a & This study \\
\hline pLR75 & $\operatorname{csg} F$ in $\mathrm{pBAD} 33$ & Nenninger et al. (2009) \\
\hline pLR92 & $\begin{array}{l}\text { a modified pBAD33 vector (modification: } \mathrm{cm}^{\mathrm{R}} \text { gene does not contain an Ncol site) containing } \\
\text { csgG-HA flanked by Sacl-Ncol }\left(5^{\prime}\right) \text { and Kpnl-Bglll-Pst1 }\left(3^{\prime}\right) \text { sites }\end{array}$ & Robinson et al. (2006) \\
\hline pLR93 & $\operatorname{csg} G$ in pLR1 & Robinson et al. (2006) \\
\hline pLR116 & the CsgAss-A22-CpxP-his Ncol-Pstl fragment of pLR50 cloned into pTrc99a & This study \\
\hline pMC1 & $\operatorname{csg} G$ in $\mathrm{p} T r c 99 \mathrm{a}$ & Chapman et al. (2002) \\
\hline pNH27 & gene encoding cytoplasmic CsgE inserted into the Ncol-BamHI sites of pET11d & This study \\
\hline
\end{tabular}

(Fig. 1A). It is possible that fibres produced in the presence of excess CsgE may have subtle biochemical differences such as CR binding capacity. When CsgF was overexpressed in the $c s g E$ mutant, no extracellular fibres of any kind could be detected by EM (data not shown) and no CsgA or CsgB protein could be detected by Western analysis, even after prolonged exposure of the blot (Fig. 1C, lane 9), which is consistent previous findings indicating that $\mathrm{CsgF}$ and $\mathrm{CsgA}$ levels are inversely correlated (Chapman et al., 2002; Nenninger et al., 2009). Similar protein profiles of all 10 strains in Fig. 1 were obtained when samples containing the bacterial lawn and the underlying agar were analysed by Western blot, indicating that the absence of CsgA, CsgB and CsgF is the result of protein instability and not due to secretion into the agar media (Fig. S1B and data not shown).
To confirm that CsgA, CsgB and CsgF were surfaceexposed in the complemented csgE strains (csgE/pE and $\mathrm{csgE} / \mathrm{pG}^{+}$) as they are in the wild-type strain (Hammar et al., 1996; Bian and Normark, 1997; Nenninger et al., 2009), we performed whole cell proteinase $K$ assays. In both of the complemented csgE strains, CsgA, CsgB and $\mathrm{CsgF}$ were susceptible to digestion by proteinase $\mathrm{K}$, while the periplasmic protein DsbA was not, indicating all three curli proteins were surface-exposed (Fig. S1A, lanes 3-6). Even the small amounts of CsgA, CsgB and CsgF protein detected in the $\operatorname{csg} E$ mutant containing an empty vector were found to be surface-exposed (Fig. S1A, lanes 1-2), suggesting that CsgE was not absolutely required for the surface localization of any of the secreted curli proteins. Collectively these data indicate that CsgE is dispensable for curli fibre formation when CsgG is overexpressed, and 


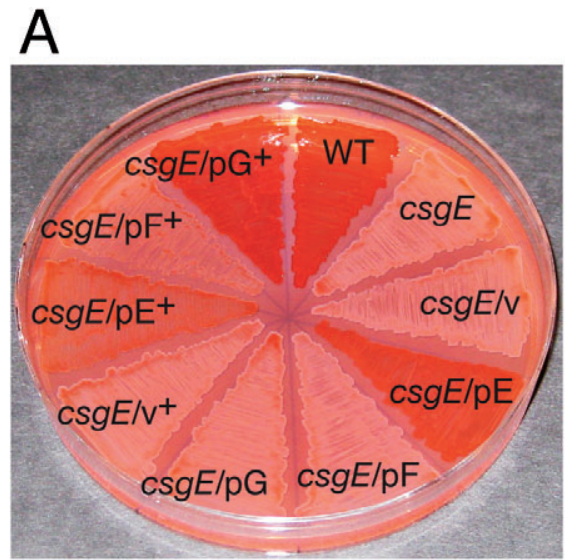

B
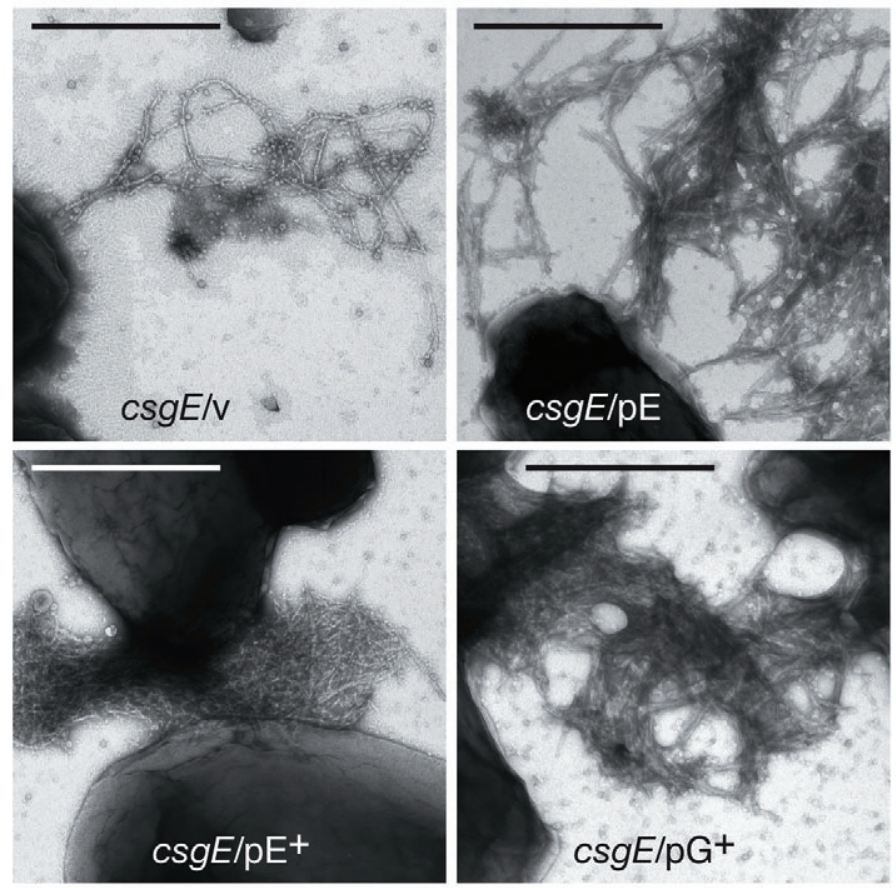

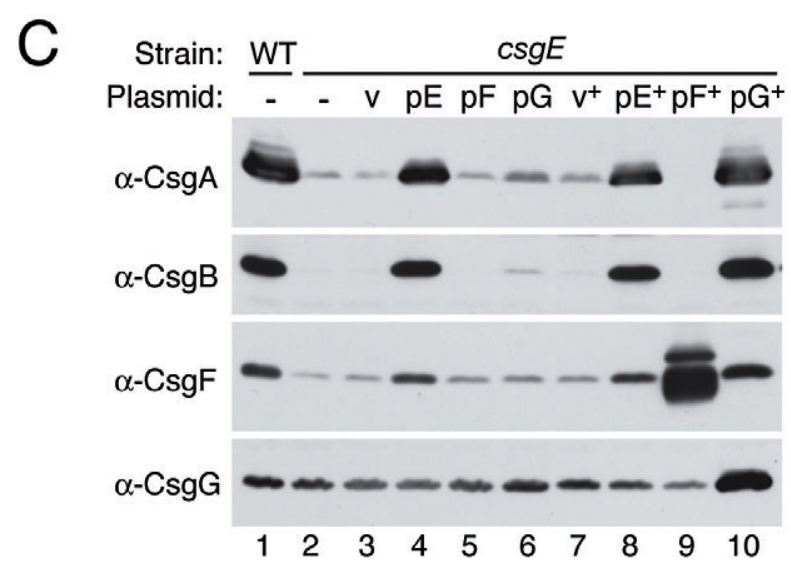

Fig. 1. Overexpression of CsgG restores curli fibre formation to the $\operatorname{csg} E$ mutant. A. The following strains were grown on YESCA-Congo red agar for $48 \mathrm{~h}$ at $26^{\circ} \mathrm{C}$ : wild-type (WT, MC4100); $\operatorname{csgE}(\mathrm{MHR} 480) ; \operatorname{csg} E / \mathrm{v}$

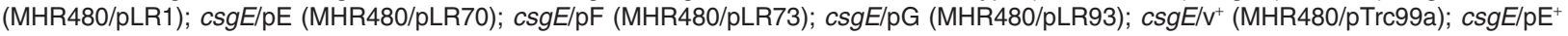
(MHR480/pLR71); csgE/pF ${ }^{+}$(MHR480/pLR74); csgE/pG ${ }^{+}$(MHR480/pMC1). The plus symbol indicates an overexpression vector.

B. Negative stain electron micrographs of strains csgE/v (MHR480/pLR1), csgE/pE (MHR480/pLR70), csgE/pE (MHR480/pLR71) and $\operatorname{csgE} / \mathrm{pG}^{+}$(MHR480/pMC1) after $48 \mathrm{~h}$ of growth on YESCA agar at $26^{\circ} \mathrm{C}$. Scale bars $=0.5 \mu \mathrm{m}$.

C. Western blot analysis of the same strains as in (A). Whole cells were collected from YESCA agar after $48 \mathrm{~h}$ of growth at $26^{\circ} \mathrm{C}$, treated with formic acid and analysed for the presence of CsgA, CsgB, CsgF and CsgG.

suggest that CsgE may play the role of an efficiency factor in the CsgG-mediated secretion of curli proteins.

\section{CsgE confers erythromycin resistance to cells overexpressing CsgG}

Our initial observations suggested that CsgE might modulate the activity of the $\mathrm{CsgG}$ pore. The pore-like properties of CsgG were previously demonstrated, in part, using antibiotic sensitivity assays. In these assays, E. coli containing an empty vector are erythromycin-resistant while E. coli overexpressing CsgG are susceptible to erythromycin (Robinson et al., 2006). To determine whether CsgE affects CsgG-mediated erythromycin sensitivity, CsgG was coexpressed with CsgE in the presence of erythromycin. As seen in Fig. 2, CsgE expression significantly increased the growth rate of bacteria overexpressing CsgG ('+ CsgE', filled squares) relative to cells 


\section{CsgE expression restores Erythromycin resistance}

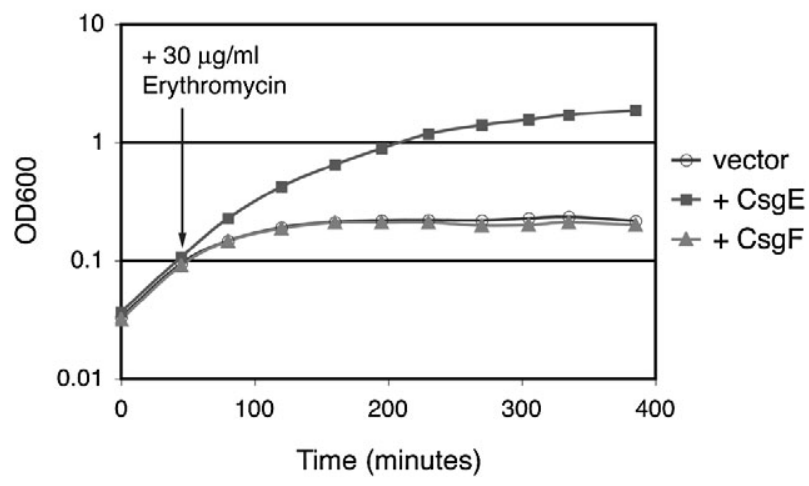

Fig. 2. Effect of CsgE on erythromycin sensitivity conferred by the overexpression of CsgG. LSR11 (MC4100 $\Delta$ csg) overexpressing CsgG from pMC1 along with an empty vector (pBAD33, open circles), CsgE (pLR42, filled squares) or CsgF (pLR58, filled triangles) were grown in $\mathrm{LB}$ shaking cultures at $37^{\circ} \mathrm{C}$ with $30 \mu \mathrm{g} \mathrm{ml}^{-1}$ erythromycin added $30 \mathrm{~min}$ after plasmid induction, and then measured by $\mathrm{OD}_{600}$ at $\sim 40$ min intervals.

expressing CsgG alone ('vector', open circles), demonstrating that coexpression of CsgE prevented CsgGdependent erythromycin sensitivity. Since CsgG levels were similar in all strains as measured by Western blot (data not shown), and we have shown previously that CsgG membrane localization is not affected by CsgE (Epstein et al., 2009), these data suggest the CsgG translocon was blocked in the presence of CsgE. Coexpression of CsgF with CsgG had no effect on erythromycin sensitivity (Fig. 2, '+ CsgF', filled triangles), suggesting the CsgG pore was in an open state in these cells and demonstrating that restoration of erythromycin resistance to CsgG-expressing cells was an activity specific to CsgE.

\section{CsgE blocks non-specific CsgG-mediated secretion}

Elucidating the concerted roles of CsgG and CsgE in curli protein secretion is complicated since all three secreted proteins (CsgA, CsgB and CsgF) are undetectable, or nearly so, in strains lacking CsgG or CsgE. Therefore, we developed a CsgG protein secretion assay using small periplasmic proteins not directly related to curli biogenesis that remain detectable in the absence of CsgG or CsgE. In this way we were able to separate the issue of protein stability from protein secretion, allowing us to track CsgGmediated secretion in the presence and absence of CsgE. In order to find proteins that were suitable CsgG secretion substrates, we screened a number of periplasmic proteins for their ability to be translocated to the supernatant in the presence of CsgG overexpression. Many proteins tested were not amenable to this assay (e.g. PhoA, DsbA, PpiA, PapD, FimC) perhaps because they are too large or tightly folded to pass through the CsgG secretion channel, which is predicted to be approximately $2 \mathrm{~nm}$ in diameter
(Stathopoulos et al., 1996; Robinson et al., 2006). However, we discovered that domain 2 of the $\mathrm{P}$ pilus chaperone, PapD (Bann and Frieden, 2004) (referred to as 'PapD2'), as well as the cpx stress response adaptor protein, CpxP (Isaac et al., 2005), were amenable for CsgG-mediated secretion. Both the PapD2 domain (93 amino acids) and CpxP (145 amino acids) are similar in size to the three secreted curli proteins (118-131 amino acids). Overexpression of CsgG from pTrc99a and PapD2 from pBAD33 in liquid cultures of the LSR11 strain (MC4100 $\Delta c s g$ ) resulted in secretion of the PapD2 protein to the supernatant (Fig. 3B, lane 2). No PapD2 was detected in the supernatant in the absence of CsgG, even after prolonged exposure of the blot, demonstrating that localization of PapD2 to the supernatant was CsgGdependent (Fig. 3B, lane 1). Similar results were obtained for the CpxP secretion construct (Fig. S2B). These data suggest that the CsgG translocon is an ungated pore under these experimental conditions, allowing secretion of certain periplasmic proteins that are not associated with curli biogenesis. The idea that overexpressed CsgG forms an ungated pore is consistent with the previous observation that CsgG overexpression renders cells sensitive to the antibiotic erythromycin (Robinson et al., 2006).

We next determined whether the addition of CsgE altered the secretion profile described above. For these

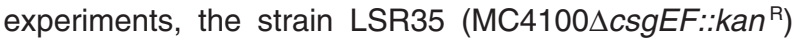
was used such that CsgG was expressed off the chromosome, while the secretion constructs (PapD2 or CpxP) and CsgE could be expressed individually from separate plasmids. Chromosomal CsgG expression in LSR35 was less than plasmid CsgG expression in LSR11 (Fig. 3D, compare lanes 2 and 4 with $5-10$ ), but was sufficient to allow secretion of PapD2 (Fig. 3C, lane 5). When CsgE was coexpressed with PapD2, PapD2 secretion to the supernatant was suppressed (Fig. 3C, compare lanes 5 and 6), indicating that CsgE can inhibit the secretion of non-curli-related proteins through the curli translocon. Similar results were obtained for the CpxP construct (Fig. S2C, lane 5). Together with the erythromycin sensitivity assay, these data suggest that CsgE provides a gating mechanism to an otherwise permissive outer membrane pore.

The N-terminus of CsgA (A22) is a curli-specific outer membrane secretion signal

Our results thus far have identified CsgE as a potential specificity factor for CsgG-mediated protein secretion, as it blocks secretion of proteins unrelated to curli biogenesis. We reasoned that the secreted curli proteins might contain a curli-specific outer membrane secretion signal that would allow secretion of curli proteins, similar to the sec signal sequence that directs proteins across the 
A

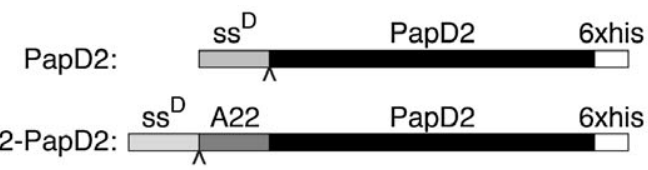

B

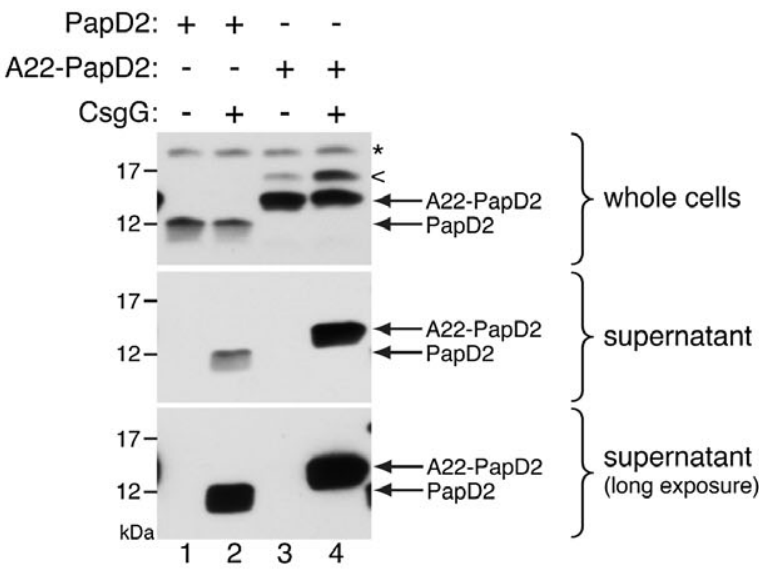

C PapD2: $+++--\cdot$

A22-PapD2: - -+++

CsgG: +++++

CsgE: -+-++

CsgF: - - + - +

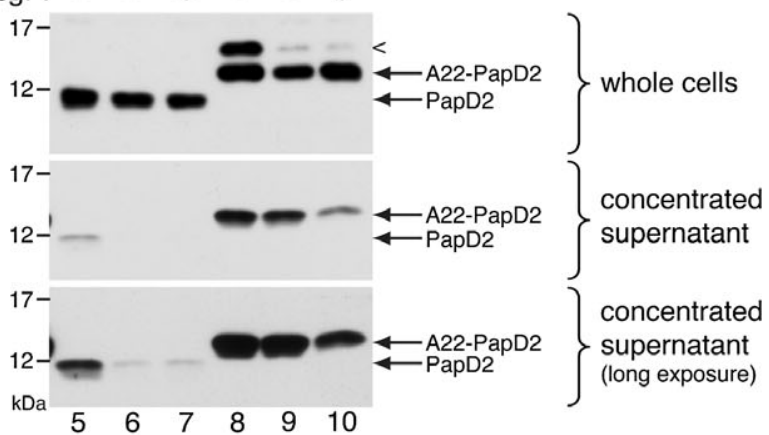

D

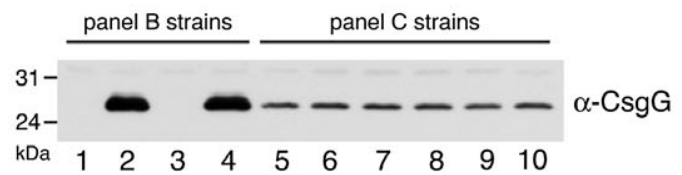

Fig. 3. Effects of CsgE on the

CsgG-dependent secretion of PapD2 and A22-PapD2.

A. A schematic diagram of the PapD2 and A22-PapD2 constructs used in the secretion assays. PapD2 consists of the 21 amino acid sec-dependent signal sequence of $\operatorname{PapD}\left(\mathrm{ss}^{\mathrm{D}}\right)$ fused to domain 2 of PapD with a C-terminal 6-his tag; domain 2 is the C-terminal 93 amino acids of PapD encompassing the second domain of this 2-domain chaperone. A22-PapD2 consists of the PapD signal sequence $\left(\mathrm{SS}^{\mathrm{D}}\right)$, the $\mathrm{N}$-terminal 22 amino acids of mature CsgA (A22), domain 2 of $\mathrm{PapD}$ and a C-terminal 6-his tag. The carat indicates the sec cleavage site.

$B$ and C. CsgG-dependent protein secretion assay: cells were grown in LB shaking cultures at $37^{\circ} \mathrm{C}$ to $\mathrm{OD}_{600} \sim 0.8$, protein expression induced for $1 \mathrm{~h}$, then whole cell and supernatant (or concentrated supernatant) fractions were subjected to Western blot analysis using PapD-specific antisera. The symbol < corresponds to the predicted size of $\mathrm{ss}^{\mathrm{D}}$-A22-PapD2 (i.e. prior to sec signal cleavage); the asterisk indicates a non-specific background band. (B) The bacterial strain LSR11 (MC4100 $\Delta c s g$ ) expressing PapD2 (lanes 1-2; pAN87) or A22-PapD2 (lanes 3-4; pAN88) and an empty vector (lanes 1 and 3; pTrc99A) or CsgG (lanes 2 and 4; pMC1). (C) The bacterial strain LSR35 (MC4100 csgEF::kan ${ }^{\mathrm{R}}$ ) expressing CsgG from the chromosome contained the following plasmids: PapD2 (lanes 5-7; pAN93) or A22-PapD2 (lanes 8-10; pAN94); and, empty vector (lanes 5 and 8; pBAD33), CsgE (lanes 6 and 9; pLR42) or CsgF (lanes 7 and 10; pLR75). $D$. Whole cell samples of the strains in (B) and $(C)$ were subjected to Western blot analysis using CsgG-specific antiseria. Lane numbers correlate to those of $(B)$ and $(C)$. inner membrane through the Sec translocon. We previously demonstrated that a fusion protein containing the $\mathrm{N}$-terminal 22-amino-acid residues of mature CsgA (A22) specifically interacts with CsgG (Robinson et al., 2006). Furthermore, A22 is not thought to participate in the core structure of curli fibres (Collinson et al., 1999), making it a prime candidate for a curli-specific secretion signal. Therefore, we created an A22 fusion protein with PapD2 (Fig. 3A) to test the hypothesis that A22 is sufficient to allow secretion past the CsgE gate and through the CsgG pore. Like PapD2, A22-PapD2 was secreted to the super- natant in a CsgG-dependent manner in LSR11 cells expressing CsgG from a plasmid (Fig. 3B, lane 4). No A22-PapD2 could be detected in the supernatant in the absence of CsgG (Fig. 3B, lane 3). A22-PapD2 was also secreted to the supernatant in LSR35 cells expressing CsgG from the chromosome (Fig. 3C, lane 8). However, in contrast to the PapD2 construct whose secretion was suppressed in the presence of CsgE, secretion of A22PapD2 was not changed with CsgE expression. The pore selectivity function is specific to $\mathrm{CsgE}$, as coexpression of CsgF decreased secretion of both PapD2 and A22-PapD2 
A

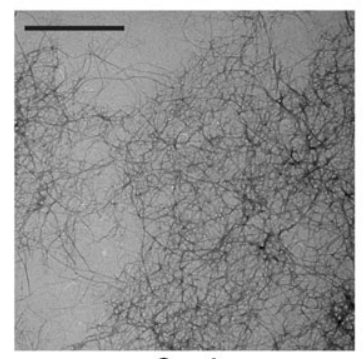

CsgA

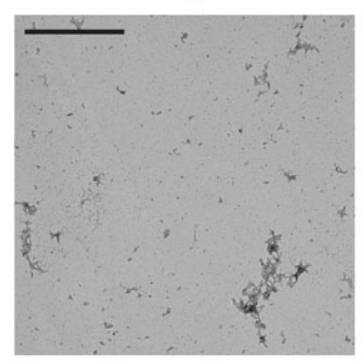

CsgA + CsgE
B

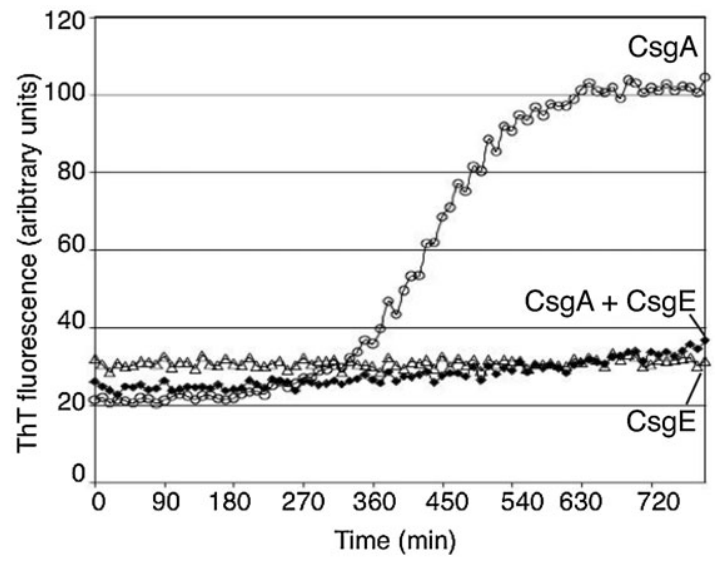

Fig. 4. CsgE prevents self-assembly of CsgA into amyloid fibres.

A. Freshly purified CsgA was incubated alone (top) or in the presence of CsgE in a near 1:1 molar ratio (bottom) for $24 \mathrm{~h}$ before visualization with transmission electron microscopy. Scale bars equal $1 \mu \mathrm{m}$. B. Real-time monitoring of CsgA self-assembly into amyloid fibres by ThT fluorescence. Approximately $25 \mu \mathrm{M}$ CsgA was incubated in reaction buffer containing ThT alone (open circles) or with $22.5 \mu \mathrm{M}$ CsgE (filled diamonds). Approximately $22.5 \mu \mathrm{M}$ CsgE was also incubated alone in ThT reaction buffer (open triangles). relative to the vector control (Fig. 3C, compare lanes 7 with 5 and 10 with 8). This result is not unexpected, as $\mathrm{CsgF}$ itself is known to be secreted through CsgG and would thus compete with other potential secretion substrates (such as PapD2 and A22-PapD2) for engagement with the CsgG secretion complex (Chapman et al., 2002; Hammer et al., 2007; Nenninger et al., 2009). As before, similar results were obtained with an A22-CpxP fusion protein (Fig. S2B and C). These data suggest that the A22 domain of CsgA is a sufficient specificity signal to allow protein secretion through a CsgE-gated CsgG pore.

\section{CsgE inhibits self-assembly of CsgA into amyloid fibres}

One outstanding question in the biosynthesis of curli fibres is how cells prevent amyloid formation on the periplasmic side of the curli assembly apparatus. The observation that CsgE provided substrate specificity to CsgG secretion raised the possibility that $\mathrm{CsgE}$ may also act on the secretion substrates themselves. Furthermore, CsgE appears to modulate the ultrastructural or biochemical properties of curli, as the absence of CsgE (csgE/v) produced fewer fibres of aberrant morphology relative to wild-type (Fig. 1B), and the overexpression of CsgE $\left(c s g E / \mathrm{pE}^{+}\right)$results in reduced $\mathrm{CR}$ binding despite having similar levels of polymerized CsgA to the $c s g E / p E$ strain (Figs $1 \mathrm{~A}$ and S1B). This may be due to interactions with, or modifications of, pre-assembled CsgA. We were unable to detect a direct protein-protein interaction between CsgA and CsgE by immunoprecipitation from cell lysates or by far-Western analysis (data not shown). However, we went on to examine the effect of CsgE on in vitro polymerization of CsgA. CsgA is soluble and unstructured immediately following purification, but after incubation for several hours CsgA begins to self-assemble into $\beta$-sheet rich amyloid fibre aggregates (Chapman et al., 2002). In vitro polymerization of CsgA can be monitored over time by the appearance of fibres by EM, and by characteristic reactivity with thioflavin $T$ (ThT). Freshly purified CsgA was incubated with or without purified $\mathrm{CsgE}$, as described in Experimental procedures. As previously shown, CsgA incubated alone polymerized into large collections of 4- to 6-nm-wide amyloid fibres (Fig. 4A, top) (Chapman et al., 2002; Wang et al., 2007). When incubated in a near 1:1 molar ratio with $\mathrm{CsgE}$, however, CsgA failed to assemble into fibres after $24 \mathrm{~h}$; only a few, small, amorphous aggregates could be detected in this reaction (Fig. 4A, bottom). CsgA polymerization with or without CsgE was also monitored by a real-time ThT fluorescence assay. A reaction containing $25 \mu \mathrm{M}$ CsgA alone exhibited exponential growth after 270 min of incubation at room temperature (Fig. 4B, open circles), while a reaction containing $25 \mu \mathrm{M}$ CsgA and $22.5 \mu \mathrm{M}$ of $\mathrm{CsgE}$ did not exhibit substantial ThT reactivity after $800 \mathrm{~min}$ of incubation (Fig. 4B, filled diamonds). Reactions containing CsgE alone showed no increase in ThT fluorescence in this assay (Fig. 4B, open triangles). As a control, strains expressing an empty vector instead of a vector containing CsgE were subjected to the same CsgE purification protocol, and the fractions corresponding to the CsgE fractions (which contain similar contaminating bands by Coomassie stain as that of a CsgE purification) were added to $25 \mu \mathrm{M}$ CsgA. This resulted in a similar ThT profile to that of CsgA alone, suggesting that 
inhibition of CsgA polymerization was CsgE specific (data not shown). Purified CsgE added to pre-formed CsgA fibres did not depolymerize CsgA into monomers, as no change in ThT fluorescence was observed for CsgA fibrils incubated with purified CsgE over a $24 \mathrm{~h}$ time period (data not shown). These results suggest that, in vitro, CsgE can prevent the self-assembly of soluble CsgA into amyloid fibres.

\section{Discussion}

While protein secretion across the inner membrane is largely carried out by the Sec channel, Gram-negative bacteria employ numerous secretion systems to translocate proteins across the outer membrane (Saier, 2006; Gerlach and Hensel, 2007; Filloux et al., 2008). During curli biogenesis, at least three curli-related proteins are translocated across the outer membrane in a CsgGdependent fashion: CsgB, CsgA and CsgF. In the absence of CsgG, these proteins are not secreted and no curli are assembled (Hammar et al., 1995; Loferer et al., 1997; Robinson et al., 2006; Nenninger et al., 2009). Likewise, csgE mutants assemble fewer curli than wild-type cells and secrete less CsgB, CsgA and CsgF (Fig. 1) (Chapman et al., 2002; Nenninger et al., 2009). Here, we found evidence that CsgE promotes efficient subunit secretion, gates the outer membrane pore and prevents aggregation of the major fibre subunit protein, CsgA.

CsgG is a limiting factor in the stability of CsgA, CsgB and $\mathrm{CsgF}$, as CsgG overexpression increases the steadystate levels of these proteins (Loferer et al., 1997; Robinson et al., 2006; Nenninger et al., 2009). We found that CsgG overexpression also suppresses the csgE mutant defect and restores the stability of all three CsgGsecreted proteins and the assembly of curli fibres (Fig. 1). Thus CsgE is required for curli fibre biogenesis when CsgG is expressed at physiological levels, but not when CsgG is artificially overexpressed. This suggests that CsgE might act as an efficiency factor for CsgG function. CsgG is functionally similar to both ushers and secretinlike proteins, which form outer membrane channels that support protein secretion to the extracellular milieu (Thanassi, 2002). Secretin-like pores are often dependent on small, periplasmic pilot proteins for their localization to the outer membrane and stability (Hardie et al., 1996a,b; Crago and Koronakis, 1998; Shevchik and Condemine, 1998). CsgE does not change CsgG steady-state levels or localization to the outer membrane (Fig. 1) (Epstein et al., 2009). Because CsgE does not modulate CsgG stability or localization to the outer membrane, its role in supporting subunit secretion is apparently different from that of secretin pilot proteins but may play a role in gating and ungating CsgG.
To better define the role of CsgE in curli protein secretion, we developed a CsgG-dependent secretion assay using non-curli proteins as secretion cargo - PapD2 and CpxP. Unlike the native curli secretion substrates (CsgA, CsgB and CsgF), PapD2 and CpxP are stable in the periplasm in the absence of CsgG or CsgE; like the curlisecreted proteins, however, both are secreted to the culture supernatant in a CsgG-dependent manner. In this way we were able to separate protein stability and protein secretion, which are inseparable for the native substrates. The secretion assays show that CsgE is able to restrict CsgG-dependent secretion of non-curli substrates while not affecting the secretion of substrates tagged with a putative curli secretion signal of CsgA, A22, suggesting that $\mathrm{CsgE}$ confers substrate selectivity to the CsgG pore (Figs 3 and S2). The A22 domain of CsgA was sufficient to provide a curli-specific signal and allow passage past the CsgE gate. However, this does not exclude the involvement of other domains of CsgA during secretion. Overexpression of A22 fusion proteins did not exhibit dominant negative activity in wild-type MC4100 cells (Fig. S3), suggesting an abundance of the A22 domain did not interfere with the secretion of wild-type curli proteins. CsgB and CsgF are also secreted to the cell surface in a CsgGdependent manner, but we have not yet identified domains in $\mathrm{CsgB}$ or $\mathrm{CsgF}$ that function in a similar manner to the A22 domain of CsgA. Further work is necessary to determine if all three known extracellular curli proteins are secreted by the same or different mechanisms.

Although overexpression of CsgG mostly suppresses the $\operatorname{csg} E$ phenotype, curli formation does not appear as efficient when compared with wild-type. When CsgG is overexpressed in a $\operatorname{csg} E$ strain, an increased proportion of unpolymerized CsgA can be detected in the underlying agar compared with wild-type cells (Fig. S1B). Furthermore, we observed less protease-resistant CsgB in the $\operatorname{csg} \mathrm{E} / \mathrm{pG}^{+}$strain compared with $\operatorname{csg} \mathrm{E} / \mathrm{pE}$ (see Fig. S1A, compare lanes 4 and 6 ). This may be an indication that less CsgB is in a protease-resistant amyloid-like conformation, and also supports the idea that a portion of curli subunits fail to polymerize in the $\operatorname{csg} \mathrm{E} / \mathrm{pG}^{+}$strain, resulting in inefficient curli formation.

The suggestion that CsgE participates in gating the outer membrane pore is supported by our finding that CsgE restores erythromycin resistance to cells rendered erythromycin sensitive by CsgG overexpression (Fig. 2). Thus, CsgE prevents non-specific translocation of small proteins and large molecules through the CsgG pore, providing a gate to an otherwise permissive outer membrane channel. Other outer membrane pores also require gating mechanisms in order to maintain membrane integrity and pore specificity, such as FepA and PapC (Liu et al., 1993; Buchanan et al., 1999; Remaut et al., 2008). The recently solved crystal structure of the PapC usher 
protein showed that PapC contains a folded plug domain that occludes the central channel to prevent non-specific translocation (Remaut et al., 2008). Conformational changes of the usher during pilus biogenesis result in a reorganization of the plug, thus ungating the pore, allowing subunit translocation across the usher (Phan et al., 2011). In a similar manner, several members of the secretin family are thought to contain plug domains that fold back into and occlude the channel and thus provide the gating mechanism (PuID, XcpQ, OutD and possibly TcpC) (Shevchik et al., 1997; Brok et al., 1999; Nouwen et al., 1999; 2000; Bose and Taylor, 2005). Thus, the CsgGCsgE pore gating mechanism represents a variation of the theme for most ushers and secretins that are selfgating. It remains possible that CsgG contains a plug or gating domain itself, but requires CsgE to fully engage this domain such that it properly functions as a selectivity barrier. An interesting comparison can be made between the CsgGE curli secretion complex and outer membrane components of the type IV bundle forming pilus (bfp) system of EPEC. Like CsgG, the BfpB secretin is a lipoprotein that does not require a pilot protein for stability or outer membrane targeting, but does have a small interacting protein, BfpG, that is not a lipoprotein. It has been proposed that BfpG functions as a gate to the $\mathrm{BfpB}$ pore, but this has not been directly tested (Schmidt et al., 2001; Daniel et al., 2006).

In addition to providing a gating mechanism, CsgE may possess a second role in curli biogenesis. We found that purified CsgE was able to prevent the polymerization of purified CsgA in an in vitro reaction. This suggests a potential chaperone-like role for $\mathrm{CsgE}$ with respect to CsgA. If biologically relevant, this activity in vivo could ensure that secretion of soluble CsgA precedes fibre formation, thereby preventing formation of periplasmic amyloid oligomers or fibres and abrogating intracellular amyloid toxicity. This activity would also promote secretion efficiency, as the CsgG pore could more easily accommodate a CsgA monomer rather than an aggregate. This is analogous to the role of the periplasmic chaperones of the chaperone-usher pathway of pilus biogenesis. Pilus chaperones cap the interactive surfaces of the subunits, to prevent non-productive interactions of subunits in the periplasm (Sauer et al., 1999; Barnhart et al., 2000; Sauer et al., 2000). Specific targeting of chaperone/subunit complexes to the usher promotes conformational changes resulting in the opening of the usher gate and translocation across the usher and assembly of subunits (Kuehn et al., 1991; Dodson et al., 1993; Bullitt et al., 1996; Jones et al., 1997). Amyloid inhibition has been described for a number of small heat shock proteins (e.g. HSP-16 and $\alpha$-crystallin) and an abundant cerebrospinal fluid protein, lipocalin-type prostaglandin D synthase, all of which inhibit aggregation of the clinically important $A \beta$ peptide involved in the pathogenesis of Alzheimer's disease (Hatters et al., 2001; Fonte et al., 2002; Kanekiyo et al., 2007; Fonte et al., 2008; Tanaka et al., 2008). While an exciting possibility, whether the activity that we observed for CsgE in vitro also occurs in vivo remains to be determined. Certainly, there are a number of bacterial periplasmic chaperones (FkpA, Skp and chaperones of chaperone-usher pathway systems) that prevent the aggregation and facilitate the folding of periplasmic proteins (Ramm and Pluckthun, 2000; Sauer et al., 2004; Walton and Sousa, 2004). It would not be surprising, then, that the curli assembly apparatus includes a protein that prevents premature interactions of a highly aggregative protein, CsgA, until it is successfully delivered to and exported through its outer membrane secretion pore, CsgG. Given the rapid and toxic nature of amyloid polymerization reactions, there are likely additional control mechanisms in place in order to prevent periplasmic curli subunit aggregation. These mechanisms could be at the transcriptional, translational or posttranslational levels. Results from reporter fusion assays show only a small decrease in $\operatorname{csg} B A$ translation in the absence of CsgG, suggesting the majority of this control occurs at the post-translational level (Loferer et al., 1997). Indeed, we have never observed periplasmic intermediates of either curli subunit, including in the csgE mutant, nor have we observed intracellular aggregates or fibres, suggesting that in the absence of secretion, curli subunits are very efficiently degraded.

In stark contrast to disease-associated amyloidogenesis that underlies such neurodegenerative aliments like Alzeihmer's and Parkinson's disease, assembly of curli fibres is accomplished in a directed manner, without associated cellular toxicity. The curli assembly machine functions to guide the secretion of its amyloidogenic subunits across the outer membrane such that fibre formation is promoted on the cell surface, and restricted in the periplasm (Hammar et al., 1996; Loferer et al., 1997; Chapman et al., 2002; Robinson et al., 2006; Hammer et al., 2007; Nenninger et al., 2009). These opposing tasks appear to be executed by the two small chaperone-like proteins of curli biogenesis, CsgE and CsgF. Future definition of the molecular mechanisms of CsgE and CsgF will increase our understanding of how amyloid formation is controlled, a biological problem that $E$. coli has solved in the form of this unique biogenesis pathway.

\section{Experimental procedures}

\section{Bacterial strains and plasmids}

Bacterial strains and plasmids used in this study can be found in Table 1. Primers used to make genetic constructs can be found in Table 2. 
Table 2. Primers used in this study.

Primer name

csgE KO F no FRT

CSgF KO R no FRT

csgA F Kpn-RBS-Nco

$\operatorname{csgA-cpxP} R$

csgA-cpxP $F$

cpxP his R Pst (LR)

csgE F Kpn-RBS-Nco

csgE R 3XS-Pst

csgE R BamHI

csgF F Nco

csgF R Pst

a22cpxP F delA22

a22cpxP R delA22

papDss(ala) Kpnl mcpxP F Ncol

cpxP his R Pstl (AN)

papDss(ala) mcsgA F Ncol

csgA22 R Kpnl

papD2 F Kpnl

papD2-his R Pstl

Sec- CsgE For Ncol

CsgE 6× His Rev BamHI
Primer sequence

AGCGGTTTCCTGGGCAAACGATAACCTCAGGCGATAAAGCCACGCTGCCGCAAGCACTC

AATAAGCGCTGCATGATTATTTTCCTTATGAAGCTGGGGAATAGGAACTTCAAGATCC

GTTTGGTACCACACAGGAAACAGACCATGGCGAAACTTTTAAAAGTAGCAGCAATTGCAGC

CGCCTGAACCGACTTCAGCATTTGGGCCGCTATTATTACC

GGTAATAATAGCGGCCCAAATGCTGAAGTCGGTTCAGGCG

GTTTCTGCAGTTAGTGGTGGTGGTGGTGGTGCTGGGAACGTGAGTTGCTAC

GTTTGGTACCACACAGGAAACAGACCATGGCGAAACGTTATTTACGCTGGATTGTGGC

GTTTCTGCAGTTATTAGATCCTTAGAATTCATCATGCGCCAAATCGCCC

GTTTGGATCCTTAGAATTCATCATGCGCCAAATCGCC

GTTTCCATGGCACGTGTCAAACATGCAGTAG

GTTTCTGCAGTTAAAAATCGGTTGAGTTATTTTGTAAACC

CGGTAGCGCTCTGGCAGCTGAAGTCGGTTC

GAACCGACTTCAGCTGCCAGAGCGCTACCG

CGAGCCATGGCGATTCGAAAAAAGATTCTGATGGCTGCCATCCCCCTGTTTGTTATATCCGGGG

CAGACGCTGGTACCGCTGAAGTCGGTTCAGGCGATAAC

CTCGCTGCAGTTAGTGGTGGTGGTGGTGGTGCTGGGAACGTGAGTTGCTACTACT

CGAGCCATGGCGATTCGAAAAAAGATTCTGATGGCTGCCATCCCCCTGTTTGTTATATCCGGGGC AGACGCTGGTGTTGTTCCTCAGTACGGCGG

CGAGGGTACCATTTGGGCCGCTATTATTACCGCCACC

CGACGGTACCGAAGTATGGCAGGACCAGTTAATTCTG

CGACCTGCAGTTAGTGGTGGTGGTGGTGGTGTTTCTCTTTTTTCACAGAGCAACGGCTAC

GCGTTTCCATGGCCGTTGAGGTAGAAGTCCCGGGA

GTTTAAAGCTTGGATCCTTAGTGATGGTGATGGTGATGGAATTCATCATGCGCCAAATC
Strain construction. LSR11 (MC4100 $\Delta c s g$ ) was created by removing the $k a n^{\mathrm{R}}$ cassette from LSR5 (MC4100 $\Delta c s g:: \mathrm{kan}^{\mathrm{R}}$ ) (Chapman et al., 2002) using FLP recombinase according to standard techniques (Datsenko and Wanner, 2000). LSR35 was constructed by deleting the csgEF locus with a PCR product encoding the $k^{2} n^{R}$ cassette from pKD13 using the phage $\lambda$ Red recombinase method (Datsenko and Wanner, 2000). The resistance cassette was amplified from pKD13 with the primers $\operatorname{csg} E$ KO F no FRT and $c s g F$ KO R no FRT.

Plasmid construction. Unless otherwise indicated, chromosomal DNA from MC4100 was used as a template in PCRs. pLR50 containing the CsgAss-A22-CpxP-his fusion was synthesized by overlap PCR. An N-terminal portion encoding residues 1-42 of premature $\mathrm{CsgA}$ and the first 20 residues of mature CpxP was amplified with the primers csgA $\mathrm{F}$ KpnRBS-Nco and csgA-cpxP R. A C-terminal region encoding residues 36-42 of CsgA and his-tagged mature CpxP was amplified with the primers csgA-cpxP F and cpxP his R Pst (LR). The two regions were fused together in a third PCR, and the product was cloned into the Kpnl-Pstl sites of pBAD33. pLR116 and pLR51 were made by subcloning the Ncol-Pstl fragment from pLR50 into pTrc99a and pLR1 respectively. pAN65 and pAN66 were made by deleting the region encoding the CsgA A22 domain using site-directed mutagenesis (Stratagene QuickChange protocol) of pLR50 and pLR116, respectively, with the primers a22cpxP $\mathrm{F}$ delA22 and a22cpxP R delA22. pAN69 was made by ligating the PCR product of primers papDss(ala) Kpnl mcpxP F Ncol and cpxP his R Pstl (AN) and template pAN66 into the Ncol-Pstl sites of pLR92. pAN70 was created by ligating the PCR product of primers papDss(ala) mcsgA F Ncol and csgA22 R Kpnl and template pLR116 into the Ncol-Kpnl sites of pAN69. pAN87 and pAN88 were made by ligating the PCR product of primers papD2 F Kpnl and papD2-his R Pstl into the Kpnl-
Pstl sites of pAN69 and pAN70 respectively. pAN93 and pAN94 were made by subcloning the Ncol-Pstl fragments of pAN87 and pAN88, respectively, into pTrc99a. pLR42 was made by ligating the PCR product of csgE F Kpn-RBS-Nco and csgE R 3XS-Pst into the Kpnl-Pstl sites of pBAD33. pLR70 was created by subcloning the Ncol-Pstl fragment of pLR42 into pLR1. pLR71 was made by ligating the PCR product of csgE F Kpn-RBS-Nco and csgE R BamHI into the Ncol-BamHI sites of pTrc99a. pLR74 was made by ligating the PCR product of csgF F Nco and csgF R Pst into the Ncol-Pstl sites of pTrc99a. pNH27 encodes cytoplasmic CsgE (lacking the sec signal sequence) with a C-terminal 6-his tag and was cloned into the Ncol-BamHI sites of pET11d using the primers Sec- CsgE For Ncol and CsgE 6x His Rev BamHI.

\section{Growth conditions}

Chromosomal curli expression was induced by growing bacteria on YESCA agar (per litre: $10 \mathrm{~g}$ Casamino acids, $1 \mathrm{~g}$ yeast extract, $20 \mathrm{~g}$ agar; for CR-YESCA agar, supplement with $50 \mu \mathrm{g} \mathrm{ml}^{-1} \mathrm{CR}$ ) for $48 \mathrm{~h}$ at $26^{\circ} \mathrm{C}$ (Fig. 1). Plasmid gene expression in liquid cultures (Figs 2, 3 and S2) was induced with IPTG $(0.1-0.5 \mathrm{mM})$ for pTrc99a-based vectors and arabinose $[0.05-0.2 \%(\mathrm{w} / \mathrm{v})]$ for pBAD33-based vectors in LB broth, shaking at $37^{\circ} \mathrm{C}$. Inducer concentrations were the same within individual experiments, except for Fig. S2B, where pLR50 strains were induced with $0.001 \%$ ara and pAN65 strains induced with $0.025 \%$ ara; this was to equalize expression between the A22-CpxP-his and CpxP-his constructs, since CpxP-his expression levels were typically lower than A22-CpxP-his. For maintenance of plasmids in broth cultures, bacteria were grown with $100 \mathrm{gg} \mathrm{ml}^{-1}$ ampicillin, and $34 \mu \mathrm{g} \mathrm{ml}^{-1}$ chloramphenicol. LSR35 cultures were further supplemented with $10 \mu \mathrm{g} \mathrm{ml}^{-1}$ kanamycin. 
Table 3. Antibodies used in this study.

\begin{tabular}{|c|c|c|c|}
\hline Antibodies & Description & $\begin{array}{l}\text { Dilution used in } \\
\text { Western blot }\end{array}$ & Source/reference \\
\hline$\alpha$-CsgA & Polyclonal rabbit antiserum to CsgA & $1: 5000-1: 10000$ & Barnhart et al. (2006) \\
\hline$\alpha$ CsgB & $\begin{array}{l}\text { Polyclonal rabbit antiserum to the peptide } \\
\text { (EGSSNRAKIDQTGDY) of CsgB }\end{array}$ & $1: 2000$ & Robinson et al. (2006) \\
\hline$\alpha-C s g F$ & Polyclonal rabbit antiserum to CsgF & $1: 5000-1: 10000$ & Nenninger et al. (2009) \\
\hline$\alpha$-CsgG & Polyclonal rabbit antiserum to CsgG & $1: 5000$ & Robinson et al. (2006) \\
\hline$\alpha$-DsbA & Polyclonal rabbit antiserum to DsbA & $1: 20000$ & $\begin{array}{l}\text { Kind gift of the James Bardwell } \\
\text { Laboratory, University of Michigan }\end{array}$ \\
\hline$\alpha-P a p D$ & Polyclonal rabbit antiserum to $\mathrm{PapD}$ & $1: 5000$ & This study \\
\hline$\alpha$-CT-His & Mouse anti-C-terminal his tag & $1: 5000$ & Invitrogen \\
\hline$\alpha$ CpxP & Polyclonal rabbit antiserum to $\mathrm{CpxP}$ & $1: 10000$ & $\begin{array}{l}\text { Kind gift of the Tracy Raivio } \\
\text { Laboratory, University of Alberta }\end{array}$ \\
\hline
\end{tabular}

\section{Electron microscopy}

Electron microscopy was performed with a JEOL 1200 EX II transmission electron microscope. For whole bacteria, cells were grown on YESCA for $48 \mathrm{~h}$ and then resuspended in PBS and fixed with $1 \%$ glutaraldehyde. Fixed cells or purified protein were applied to Formvar-coated copper grids for 1-2 min. Specimens were then stained with $0.2 \%$ uranyl acetate for 1-2 min prior to visualization.

\section{Western blot analysis}

Sample preparation. For whole cell Western blot in Fig. 1, bacteria were scraped from YESCA plates, resuspended in PBS and normalized by $\mathrm{OD}_{600}$. A cell suspension volume corresponding to one optical density unit $(\mathrm{ODU}=1 \mathrm{ml}$ of $\mathrm{OD}_{600}=1.0$ ) was collected for each sample (i.e. whole cells + PBS, so that no protein was lost by pelleting of the bacteria and aspiration of PBS). For samples not pre-treated with formic acid (FA), cell suspensions were brought to $200 \mu \mathrm{l}$ using SDS loading buffer. For FA-treated samples, FA was added to cell suspensions (final $=70 \%$ or greater $F A$ ), the acid evaporated in a vacuum centrifuge, the pellet resuspended in $200 \mu \mathrm{I}$ SDS sample buffer and $\mathrm{pH}$ adjusted with $1 \mathrm{~N}$ $\mathrm{NaOH}$, if necessary. For plug samples (Fig. S1B), a circular plug $(\mathrm{d}=8 \mathrm{~mm})$ was cut from the agar and collected. +/- FA treatment of plugs was as above for whole cells, except that FA-treated plugs were solubilized in $100 \mu \mathrm{l}$ of $96 \%$ FA. All other samples for Western blot were prepared as indicated.

Western blotting. All samples were boiled for $5 \mathrm{~min}$ prior to SDS-PAGE in $15 \%$ acrylamide gels; resolved proteins were transferred to nitrocellulose membrane at $4^{\circ} \mathrm{C}$ in $25 \mathrm{mM}$ CAPS, pH 11.2 (50 $\mathrm{V}$ for $3 \mathrm{~h}$ or $12 \mathrm{~V}$ overnight).

Blocking. RT $2 \mathrm{~h}$ with rocking in $1 \times$ TBST, $1.5 \%$ milk, $1.5 \%$ BSA. Primary antibodies and their respective dilutions can be found in Table 3 . Horseradish peroxidase conjugated secondary antibody (Pierce): RT $1 \mathrm{~h}$ at 1:5000-1:10 000 dilution in blocking buffer.

Detection. Supersignal West Femto chemiluminescent substrate (Pierce). The PapD antibody was raised in rabbits against purified wild-type PapD protein (SigmaGenosys custom antisera production).

\section{Whole cell proteinase $K$ treatment}

Intact cells were scraped from YESCA agar, normalized by $\mathrm{OD}_{600}$ and two ODUs of each strain collected, as described in Western blot analysis. Two ODUs of cells were brought to $270 \mu \mathrm{l}$ with $1 \times$ PBS; $30 \mu \mathrm{l}$ of water or a $10 \times$ protease stock $\left(1 \mathrm{mg} \mathrm{ml}^{-1}\right.$ proteinase $\left.\mathrm{K}\right)$ in water was then added and vortexed to begin the reaction. After incubation for $2 \mathrm{~h}$ at $37^{\circ} \mathrm{C}$, the reaction was quenched with $2 \mathrm{mM}$ PMSF. Cells were pelleted; cell pellets were treated with $100 \mu \mathrm{l} 96 \%$ FA and prepared for immunoblotting as described in Western blot analysis.

\section{Antibiotic sensitivity assay}

Escherichia coli strain LSR11 containing pMC1 (csgG in pTrc99a) and pBAD33 (vector), pLR42 (csgE in pBAD33) or pLR58 (csgF in pBAD33) was diluted 1:500 from overnight cultures and grown to an $\mathrm{OD}_{600}$ of 0.05 in $\mathrm{LB}$ broth at $37^{\circ} \mathrm{C}$ shaking. Plasmid gene expression was induced with $0.1 \mathrm{mM}$ IPTG and $0.04 \%$ arabinose. Thirty minutes later, $30 \mu \mathrm{g} \mathrm{ml}^{-1}$ of erythromycin was added to the media. $\mathrm{OD}_{600}$ measurements were then recorded at 40 min intervals for $6 \mathrm{~h}$.

\section{CsgG-dependent secretion assays}

After growth and induction (described in Growth conditions) of the indicated strains, cultures were normalized by $\mathrm{OD}_{600}$ and whole cell pellet and supernatant samples taken for Western blot analysis. For the secretion assay presented in Figs $3 \mathrm{C}$ and $\mathrm{S} 2 \mathrm{C}$, supernatants were concentrated as follows: supernatants were obtained by pelleting cells (3600 r.p.m. $10 \mathrm{~min}$ ), then filter-sterilizing $(0.22 \mu \mathrm{m})$ the spent media; a metal affinity resin (Clontech, Talon) was added to the filter-sterilized supernatant and allowed to incubate overnight at $4^{\circ} \mathrm{C}$ with rocking; the resin was then collected, washed $3 \times$ in $1 \times$ PBS buffer, resuspended in SDS sample buffer and boiled for 5 min prior to gel loading. Filtered supernatant volumes used for the pull-down were also normalized by $\mathrm{OD}_{600}$ of the corresponding induced culture. 


\section{Purification of CsgE}

CsgE-his was expressed from pNH27 in strain NEB 3016 with $100 \mu \mathrm{g} \mathrm{ml}^{-1}$ ampicillin. Cells were grown to late log phase and induced for $1 \mathrm{~h}$ with $0.5 \mathrm{mM}$ IPTG. Cells were harvested by centrifugation at $5000 \mathrm{~g}$ for $20 \mathrm{~min}$ and resuspended in lysis buffer $(50 \mathrm{mM} \mathrm{KPi} \mathrm{pH} \mathrm{7.3,} 100 \mathrm{mM} \mathrm{NaCl}$, $10 \mathrm{mM}$ 2-mercaptoethanol, 0.1\% Tween-20, $1 \mathrm{U} \mathrm{ml}^{-1}$ DNase, $1 \mathrm{mM}$ PMSF). Cells were lysed using a French press. The lysate was centrifuged at $10000 \mathrm{~g}$ for $20 \mathrm{~min}$. The supernatant was incubated with HIS-Select HF Nickel Affinity Gel (Sigma, H0527) rotating at $4^{\circ} \mathrm{C}$ for $1 \mathrm{~h}$ and applied to a $5 \mathrm{ml}$ column (Thermo Scientific, 29922). The column was washed with lysis buffer, followed by $12.5 \mathrm{mM}$ Imidazole in $50 \mathrm{mM} \mathrm{KPi}$ $\mathrm{pH}$ 7.3. CsgE-his was eluted with $250 \mathrm{mM}$ imidazole in $50 \mathrm{mM} \mathrm{KPi} \mathrm{pH} 7.3$ and then dialysed overnight at $4^{\circ} \mathrm{C}$ against $50 \mathrm{mM} \mathrm{KPi} \mathrm{pH} \mathrm{7.3,} 100 \mathrm{mM} \mathrm{NaCl}, 1 \mathrm{mM}$ PMSF. Precipitated proteins were removed by centrifugation. The same expression and purification strategy was carried out for NEB 3016 containing an empty expression vector (pET11d), and the dialysed eluate from this purification was used as a negative control for the CsgA polymerization assay (see Real-time CsgA polymerization assay).

\section{Real-time CsgA polymerization assay}

Experiments were carried out as previously described (Hammer et al., 2007; Wang et al., 2007), except that purified CsgE-his or a negative control (see Purification of CsgE) were added to freshly purified, monomeric CsgA prior to monitoring of thioflavin T fluorescence.

\section{Acknowledgements}

We are extremely grateful to Julia Wong and Tracy Raivio (University of Alberta) for providing purified CpxP antisera, to the Bardwell laboratory for providing the DsbA antisera and to members of the Hultgren and Chapman labs for the helpful discussions during the writing of this manuscript. This work was supported by NIH Al48689 (S.J.H.) and NIH AI073847 (M.R.C.).

\section{References}

Bann, J.G., and Frieden, C. (2004) Folding and domaindomain interactions of the chaperone PapD measured by 19F NMR. Biochemistry 43: 13775-13786.

Barnhart, M.M., and Chapman, M.R. (2006) Curli biogenesis and function. Annu Rev Microbiol 60: 131-147.

Barnhart, M.M., Pinkner, J.S., Soto, G.E., Sauer, F.G., Langermann, S., Waksman, G., et al. (2000) PapD-like chaperones provide the missing information for folding of pilin proteins. Proc Natl Acad Sci USA 97: 7709-7714.

Barnhart, M.M., Lynem, J., and Chapman, M.R. (2006) GlcNAc-6P levels modulate the expression of Curli fibers by Escherichia coli. J Bacteriol 188: 5212-5219.

Bian, Z., and Normark, S. (1997) Nucleator function of CsgB for the assembly of adhesive surface organelles in Escherichia coli. EMBO J 16: 5827-5836.

Bose, N., and Taylor, R.K. (2005) Identification of a TcpCTcpQ outer membrane complex involved in the biogenesis of the toxin-coregulated pilus of Vibrio cholerae. J Bacteriol 187: 2225-2232.

Brok, R., Van Gelder, P., Winterhalter, M., Ziese, U., Koster, A.J., de Cock, H., et al. (1999) The C-terminal domain of the Pseudomonas secretin $\mathrm{XcpQ}$ forms oligomeric rings with pore activity. J Mol Biol 294: 1169-1179.

Buchanan, S.K., Smith, B.S., Venkatramani, L., Xia, D., Esser, L., Palnitkar, M., et al. (1999) Crystal structure of the outer membrane active transporter FepA from Escherichia coli. Nat Struct Biol 6: 56-63.

Bullitt, E., Jones, C.H., Striker, R., Soto, G., JacobDubuisson, F., Pinkner, J., et al. (1996) Development of pilus organelle subassemblies in vitro depends on chaperone uncapping of a beta zipper. Proc Natl Acad Sci USA 93: $12890-12895$.

Casadaban, M.J. (1976) Transposition and fusion of the lac genes to selected promoters in Escherichia coli using bacteriophage lambda and Mu. J Mol Biol 104: 541-555.

Chapman, M.R., Robinson, L.S., Pinkner, J.S., Roth, R., Heuser, J., Hammar, M., et al. (2002) Role of Escherichia coli curli operons in directing amyloid fiber formation. Science 295: 851-855.

Collinson, S.K., Parker, J.M., Hodges, R.S., and Kay, W.W. (1999) Structural predictions of AgfA, the insoluble fimbrial subunit of Salmonella thin aggregative fimbriae. $\mathrm{J} \mathrm{Mol} \mathrm{Biol}$ 290: 741-756.

Crago, A.M., and Koronakis, V. (1998) Salmonella InvG forms a ring-like multimer that requires the $\mathrm{InvH}$ lipoprotein for outer membrane localization. Mol Microbiol 30: 47-56.

Daniel, A., Singh, A., Crowther, L.J., Fernandes, P.J., Schreiber, W., and Donnenberg, M.S. (2006) Interaction and localization studies of enteropathogenic Escherichia coli type IV bundle-forming pilus outer membrane components. Microbiology 152: 2405-2420.

Datsenko, K.A., and Wanner, B.L. (2000) One-step inactivation of chromosomal genes in Escherichia coli K-12 using PCR products. Proc Natl Acad Sci USA 97: 66406645.

Dodson, K.W., Jacob-Dubuisson, F., Striker, R.T., and Hultgren, S.J. (1993) Outer-membrane PapC molecular usher discriminately recognizes periplasmic chaperone-pilus subunit complexes. Proc Natl Acad Sci USA 90: 36703674.

Epstein, E.A., Reizian, M.A., and Chapman, M.R. (2009) Spatial clustering of the curlin secretion lipoprotein requires curli fiber assembly. J Bacteriol 191: 608-615.

Filloux, A., Hachani, A., and Bleves, S. (2008) The bacterial type VI secretion machine: yet another player for protein transport across membranes. Microbiology 154: 15701583.

Fonte, V., Kapulkin, V., Taft, A., Fluet, A., Friedman, D., and Link, C.D. (2002) Interaction of intracellular beta amyloid peptide with chaperone proteins. Proc Natl Acad Sci USA 99: 9439-9444.

Fonte, V., Kipp, D.R., Yerg, J., 3rd, Merin, D., Forrestal, M., Wagner, E., et al. (2008) Suppression of in vivo betaamyloid peptide toxicity by overexpression of the HSP-16.2 small chaperone protein. J Biol Chem 283: 784-791.

Gerlach, R.G., and Hensel, M. (2007) Protein secretion systems and adhesins: the molecular armory of Gramnegative pathogens. Int J Med Microbiol 297: 401-415. 
Gibson, D.L., White, A.P., Rajotte, C.M., and Kay, W.W. (2007) AgfC and AgfE facilitate extracellular thin aggregative fimbriae synthesis in Salmonella enteritidis. Microbiology 153: 1131-1140.

Guzman, L.M., Belin, D., Carson, M.J., and Beckwith, J. (1995) Tight regulation, modulation, and high-level expression by vectors containing the arabinose PBAD promoter. J Bacteriol 177: 4121-4130.

Hammar, M., Arnqvist, A., Bian, Z., Olsen, A., and Normark, S. (1995) Expression of two csg operons is required for production of fibronectin- and congo red-binding curli polymers in Escherichia coli K-12. Mol Microbiol 18: 661-670.

Hammar, M., Bian, Z., and Normark, S. (1996) Nucleatordependent intercellular assembly of adhesive curli organelles in Escherichia coli. Proc Natl Acad Sci USA 93: 6562-6566.

Hammer, N.D., Schmidt, J.C., and Chapman, M.R. (2007) The curli nucleator protein, CsgB, contains an amyloidogenic domain that directs CsgA polymerization. Proc Natl Acad Sci USA 104: 12494-12499.

Hardie, K.R., Lory, S., and Pugsley, A.P. (1996a) Insertion of an outer membrane protein in Escherichia coli requires a chaperone-like protein. EMBO J 15: 978-988.

Hardie, K.R., Seydel, A., Guilvout, I., and Pugsley, A.P. (1996b) The secretin-specific, chaperone-like protein of the general secretory pathway: separation of proteolytic protection and piloting functions. Mol Microbiol 22: 967-976.

Hatters, D.M., Lindner, R.A., Carver, J.A., and Howlett, G.J. (2001) The molecular chaperone, alpha-crystallin, inhibits amyloid formation by apolipoprotein C-II. J Biol Chem 276: 33755-33761.

Isaac, D.D., Pinkner, J.S., Hultgren, S.J., and Silhavy, T.J. (2005) The extracytoplasmic adaptor protein CpxP is degraded with substrate by DegP. Proc Natl Acad Sci USA 102: 17775-17779.

Jones, C.H., Danese, P.N., Pinkner, J.S., Silhavy, T.J., and Hultgren, S.J. (1997) The chaperone-assisted membrane release and folding pathway is sensed by two signal transduction systems. EMBO J 16: 6394-6406.

Kanekiyo, T., Ban, T., Aritake, K., Huang, Z.L., Qu, W.M., Okazaki, I., et al. (2007) Lipocalin-type prostaglandin D synthase/beta-trace is a major amyloid beta-chaperone in human cerebrospinal fluid. Proc Natl Acad Sci USA 104: 6412-6417.

Kuehn, M.J., Normark, S., and Hultgren, S.J. (1991) Immunoglobulin-like PapD chaperone caps and uncaps interactive surfaces of nascently translocated pilus subunits. Proc Natl Acad Sci USA 88: 10586-10590.

Liu, J., Rutz, J.M., Feix, J.B., and Klebba, P.E. (1993) Permeability properties of a large gated channel within the ferric enterobactin receptor, FepA. Proc Natl Acad Sci USA 90: 10653-10657.

Loferer, H., Hammar, M., and Normark, S. (1997) Availability of the fibre subunit CsgA and the nucleator protein CsgB during assembly of fibronectin-binding curli is limited by the intracellular concentration of the novel lipoprotein CsgG. Mol Microbiol 26: 11-23.

Nenninger, A.A., Robinson, L.S., and Hultgren, S.J. (2009) Localized and efficient curli nucleation requires the chaperone-like amyloid assembly protein CsgF. Proc Natl Acad Sci USA 106: 900-905.
Nilsson, M.R. (2004) Techniques to study amyloid fibril formation in vitro. Methods 34: 151-160.

Nouwen, N., Ranson, N., Saibil, H., Wolpensinger, B., Engel, A., Ghazi, A., and Pugsley, A.P. (1999) Secretin PulD: association with pilot PulS, structure, and ion- conducting channel formation. Proc Natl Acad Sci USA 96: 81738177.

Nouwen, N., Stahlberg, H., Pugsley, A.P., and Engel, A. (2000) Domain structure of secretin PuID revealed by limited proteolysis and electron microscopy. EMBO J 19: 2229-2236.

Olsen, A., Jonsson, A., and Normark, S. (1989) Fibronectin binding mediated by a novel class of surface organelles on Escherichia coli. Nature 338: 652-655.

Olsen, A., Wick, M.J., Morgelin, M., and Bjorck, L. (1998) Curli, fibrous surface proteins of Escherichia coli, interact with major histocompatibility complex class I molecules. Infect Immun 66: 944-949.

Phan, G., Remaut, H., Wang, T., Allen, W.J., Lebedev, A., Pirker, K.F., et al. (2011) Crystal structure of the FimD usher bound to its cognate FimC:FimH substrate. Nature (in press).

Ramm, K., and Pluckthun, A. (2000) The periplasmic Escherichia coli peptidylprolyl cis,trans-isomerase FkpA. II. Isomerase-independent chaperone activity in vitro. $J$ Biol Chem 275: 17106-17113.

Remaut, H., Tang, C., Henderson, N.S., Pinkner, J.S., Wang, T., Hultgren, S.J., et al. (2008) Fiber formation across the bacterial outer membrane by the chaperone/usher pathway. Cell 133: 640-652.

Robinson, L.S., Ashman, E.M., Hultgren, S.J., and Chapman, M.R. (2006) Secretion of curli fibre subunits is mediated by the outer membrane-localized CsgG protein. Mol Microbiol 59: $870-881$.

Romling, U., Bian, Z., Hammar, M., Sierralta, W.D., and Normark, S. (1998) Curli fibers are highly conserved between Salmonella typhimurium and Escherichia coli with respect to operon structure and regulation. J Bacteriol 180: 722-731.

Saier, M.H., Jr (2006) Protein secretion and membrane insertion systems in gram-negative bacteria. J Membr Biol 214: 75-90.

Salgado, P.S., Taylor, J.D., Cota, E., and Matthews, S.J. (2011) Extending the usability of the phasing power of diselenide bonds: SeCys SAD phasing of CsgC using a non-auxotrophic strain. Acta Crystallogr D Biol Crystallogr 67: 8-13.

Sauer, F.G., Futterer, K., Pinkner, J.S., Dodson, K.W., Hultgren, S.J., and Waksman, G. (1999) Structural basis of chaperone function and pilus biogenesis. Science 285: 1058-1061.

Sauer, F.G., Barnhart, M., Choudhury, D., Knight, S.D., Waksman, G., and Hultgren, S.J. (2000) Chaperoneassisted pilus assembly and bacterial attachment. Curr Opin Struct Biol 10: 548-556.

Sauer, F.G., Remaut, H., Hultgren, S.J., and Waksman, G. (2004) Fiber assembly by the chaperone-usher pathway. Biochim Biophys Acta 1694: 259-267.

Schmidt, S.A., Bieber, D., Ramer, S.W., Hwang, J., Wu, C.Y., and Schoolnik, G. (2001) Structure-function analysis of $\mathrm{BfpB}$, a secretin-like protein encoded by the bundle- 
forming-pilus operon of enteropathogenic Escherichia coli. J Bacteriol 183: 4848-4859.

Shevchik, V.E., and Condemine, G. (1998) Functional characterization of the Erwinia chrysanthemi OutS protein, an element of a type II secretion system. Microbiology 144: 3219-3228.

Shevchik, V.E., Robert-Baudouy, J., and Condemine, G. (1997) Specific interaction between OutD, an Erwinia chrysanthemi outer membrane protein of the general secretory pathway, and secreted proteins. EMBO J 16: 30073016.

Stathopoulos, C., Georgiou, G., and Earhart, C.F. (1996) Characterization of Escherichia coli expressing an Lpp'OmpA(46-159)-PhoA fusion protein localized in the outer membrane. Appl Microbiol Biotechnol 45: 112-119.

Tanaka, N., Tanaka, R., Tokuhara, M., Kunugi, S., Lee, Y.F., and Hamada, D. (2008) Amyloid fibril formation and chaperone-like activity of peptides from alphaA-crystallin. Biochemistry 47: 2961-2967.

Thanassi, D.G. (2002) Ushers and secretins: channels for the secretion of folded proteins across the bacterial outer membrane. J Mol Microbiol Biotechnol 4: 11-20.

Vidal, O., Longin, R., Prigent-Combaret, C., Dorel, C., Hooreman, M., and Lejeune, P. (1998) Isolation of an Escheri- chia coli K-12 mutant strain able to form biofilms on inert surfaces: involvement of a new ompR allele that increases curli expression. J Bacteriol 180: 2442-2449.

Walton, T.A., and Sousa, M.C. (2004) Crystal structure of Skp, a prefoldin-like chaperone that protects soluble and membrane proteins from aggregation. Mol Cell 15: 367374.

Wang, X., Smith, D.R., Jones, J.W., and Chapman, M.R. (2007) In vitro polymerization of a functional Escherichia coli amyloid protein. J Biol Chem 282: 3713-3719.

Zogaj, X., Bokranz, W., Nimtz, M., and Romling, U. (2003) Production of cellulose and curli fimbriae by members of the family Enterobacteriaceae isolated from the human gastrointestinal tract. Infect Immun 71: 4151-4158.

\section{Supporting information}

Additional supporting information may be found in the online version of this article.

Please note: Wiley-Blackwell are not responsible for the content or functionality of any supporting materials supplied by the authors. Any queries (other than missing material) should be directed to the corresponding author for the article. 